\title{
Nornes Características da epidemia de dengue em Pinhalzinho, Santa Catarina, 2015-2016*
}

doi: $10.5123 / 51679-49742020000400007$

\author{
Characteristics of the dengue epidemic in Pinhalzinho, Santa Catarina, Brazil, 2015-2016
}

Características de la epidemia de dengue en Pinhalzinho, Santa Catarina, Brasil, 2015-2016

\author{
Denise Catarina Andrioli ${ }^{1}$ - (1) orcid.org/0000-0002-2173-3014 \\ Maria Assunta Busato ${ }^{1}$ - (1) orcid.org/0000-0003-0043-7037 \\ Junir Antonio Lutinski ${ }^{1}$ - (1) orcid.org/0000-0003-0149-5415 \\ ${ }^{1}$ Universidade Comunitária da Região de Chapecó, Programa de Pós-Graduação em Ciências da Saúde, Chapecó, SC, Brasil
}

\section{Resumo}

Objetivo: Descrever as características da epidemia da dengue em Pinhalzinho, Santa Catarina, Brasil, e investigar os efeitos das variáveis climáticas sobre a infestação pelo mosquito Aedes aegypti. Métodos: Estudo ecológico, com dados sobre casos de dengue, hospitalizações e óbitos pela doença, em 2015 e 2016, além das variáveis meteorológicas e focos de Ae. aegypti, de 2015 a 2018. Resultados: No período epidêmico de 2015-2016, a taxa de incidência de dengue foi de 12.695,2/100 mil habitantes. Foi registrada maior incidência no sexo feminino (13.926,4/100 mil hab.) e nas faixas etárias acima de 50 anos (17.162,0/100 mil hab.). A temperatura média e a umidade relativa do ar apresentaram relação positiva com o aumento de focos de Ae. aegypti. Conclusão: A incidência de dengue durante a epidemia foi a maior já registrada no país. As condições climáticas devem ser consideradas no planejamento das ações de controle vetorial e prevenção à dengue.

Palavras-chave: Aedes; Clima; Epidemiologia Descritiva.

*Artigo derivado da dissertação de Mestrado em Ciências da Saúde, de autoria de Denise Catarina Andrioli, intitulada 'Avaliação da Epidemia de Dengue em Pinhalzinho/SC', defendida junto ao Programa de Pós-Graduação em Ciências da Saúde da Universidade Comunitária da Região de Chapecó (Unochapecó) em 2019.

Endereço para correspondência:

Denise Catarina Andrioli - Rua Henrique Dias, 253 D, Passo dos Fortes, Chapecó, SC, Brasil. CEP: 89805-075

E-mail: deniseandrioli@unochapeco.edu.br 


\section{Introdução}

A infecção causada, responsável por cerca de 390 milhões de infecções a cada ano, é considerada um dos maiores problemas de Saúde Pública no mundo.$^{1,2} \mathrm{~A}$ mesma avaliação serve para o Brasil, onde novos desafios são impostos pela introdução do vírus chikungunya em 2014 e do vírus Zika em 2015, ${ }^{3}$ e pela persistência e crescente número de casos graves e óbitos causados pela dengue a partir de $2010 .{ }^{4}$

Santa Catarina foi o último estado do Brasil a registrar casos autóctones de dengue, em 2011. ${ }^{5} \mathrm{Em} 2015$, houve 0 registro da primeira epidemia de dengue no estado, no município de Itajaí. A identificação e a avaliação das epidemias de dengue são importantes para subsidiar a adoção de estratégias mais eficientes de controle da doença e prevenção de novas epidemias.

\section{Santa Catarina foi o último estado do Brasil a registrar casos autóctones de dengue, em 2011. Em 2015, houve o registro da primeira epidemia de dengue no estado, no município de Itajaí.}

A partir dessa compreensão, 0 estudo teve 0 objetivo de descrever as características da epidemia da dengue no município catarinense de Pinhalzinho, ocorrida entre 2015 e 2016, e investigar os efeitos das variáveis climáticas sobre o número de focos do mosquito Aedes aegypti naquela localidade, no período de 2015 a 2018.

\section{Métodos}

Foi realizado estudo ecológico no município de Pinhalzinho, situado na região oeste do estado de Santa

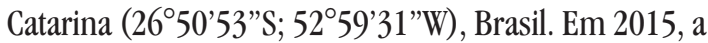
população local era de 18.700 habitantes, dos quais $84 \%$ residentes na área urbana. Pinhalzinho apresenta um clima subtropical, índice de desenvolvimento humano (IDH) de 0,783 e 94,9\% de seus domicílios contam com esgotamento sanitário adequado, de acordo com último Censo Demográfico, realizado em $2010{ }^{6}$

Os dados das notificações dos casos de dengue no período de 2015 a 2016 e hospitalizações relacionadas foram obtidos junto à Secretaria de Saúde do município, por meio do Sistema de Informação de Agravo de Notificação (Sinan) e do sistema TABNET do Departamento de Informática do Sistema Único de Saúde (Datasus).
As taxas de incidência foram calculadas segundo faixas etárias e sexo, para grupos de 100 mil habitantes. Para verificação de possíveis associações entre as variáveis nominais, utilizou-se o teste ${ }^{2}$ (qui-quadrado), com nível de significância de 5\%.

Os dados meteorológicos, referentes ao período de julho de 2015 a dezembro de 2018, foram obtidos junto ao Centro de Informações de Recursos Ambientais e de Hidrometeorologia de Santa Catarina. Os dados referentes à evolução da infestação do mosquito Ae. aegypti foram obtidos junto à Diretoria de Vigilância Epidemiológica de Santa Catarina. 0 período do estudo foi definido para se conhecer o comportamento do vetor diante das variáveis climáticas, durante e após a epidemia. Foram utilizadas análises descritivas de frequências, análise bruta e de regressão linear múltipla (nível de significância: $p<0,05)$.

Os dados foram tabulados com uso do software Excel, versão 2016. Para as análises estatísticas, utilizou-se 0 software SPSS, versão 20.0.

0 projeto da pesquisa foi aprovado pelo Comitê de Ética em Pesquisa envolvendo Seres Humanos, sob Parecer $\mathrm{n}^{0}$ 2.633.107, em 3 de maio de 2018.

\section{Resultados}

Entre 2015 e 2016, o município de Pinhalzinho enfrentou uma epidemia de dengue, com 2.374 casos notificados (DENV-1) e taxa de incidência de 12.695,2 casos por 100 mil habitantes. Houve um óbito associado à doença, no período.

A maioria dos casos notificados $(54,3 \%)$ foi do sexo feminino, com taxa de incidência de 13.926,4/100 mil habitantes. A média da idade dos casos foi de 38 anos $(\mathrm{DP} \pm 20)$ - idade mínima de zero e máxima de 103 anos -, sendo a faixa etária mais acometida a dos 20 aos 29 anos. Observou-se maior incidência de casos nas faixas etárias mais elevadas (Figura 1).

Em 2015, foram confirmados quatro casos de dengue, um em novembro (importado) e três no mês de dezembro (autóctones).

Em 2016, foram confirmados 2.370 casos autóctones (Figura 2). Nesse período foram registradas 130 hospitalizações por dengue no município. 0 maior número de casos foi registrado em março de 2016, com 53 internações (41\% das internações no ano), seguido por fevereiro (34 internações, ou 26\% das internações no ano) e abril (22 internações; $17 \%$ ) do mesmo ano. 


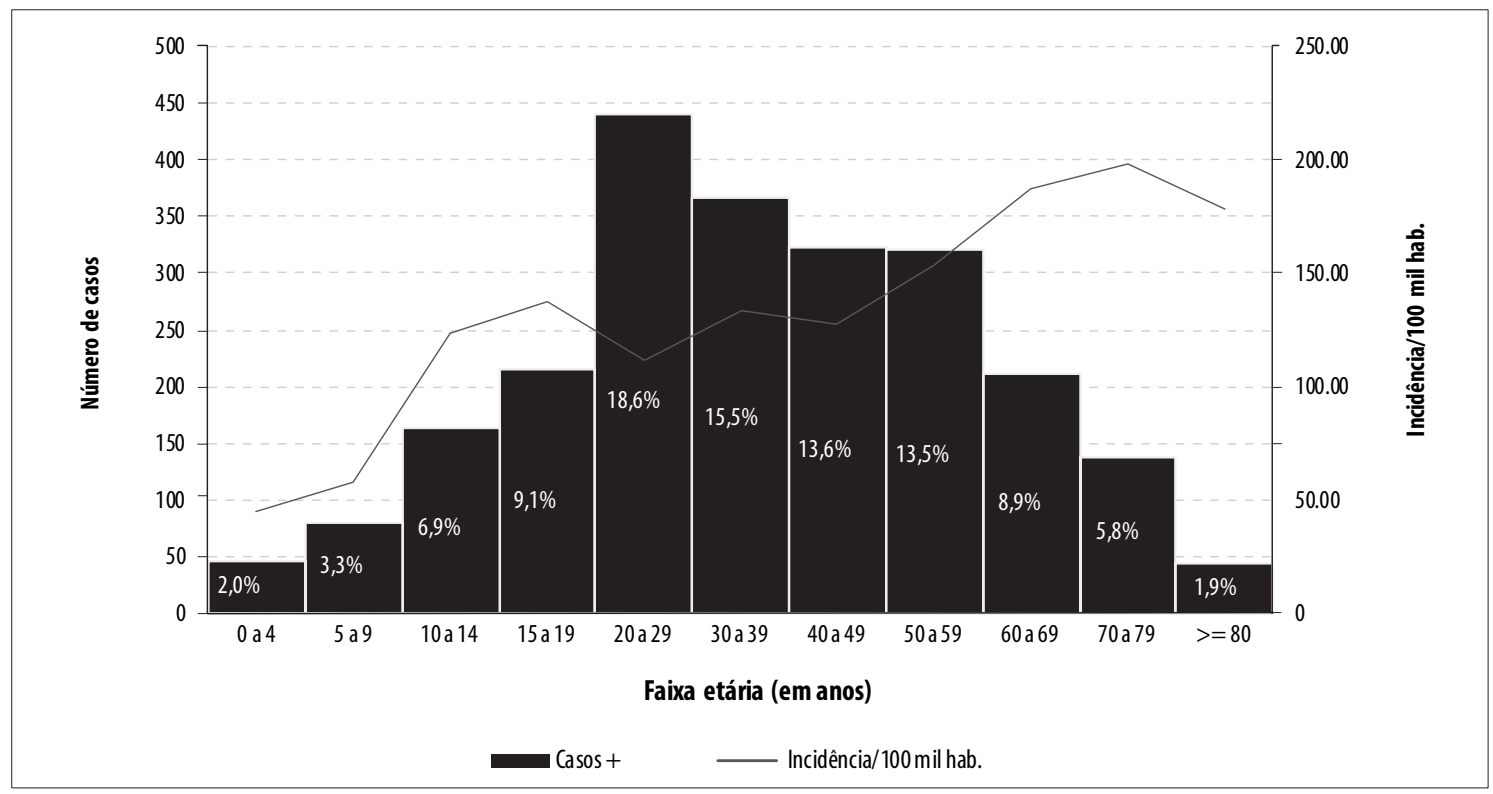

Figura 1 - Distribuição do número de casos e da incidência de dengue por faixa etária, Pinhalzinho, Santa Catarina, 2015-2016

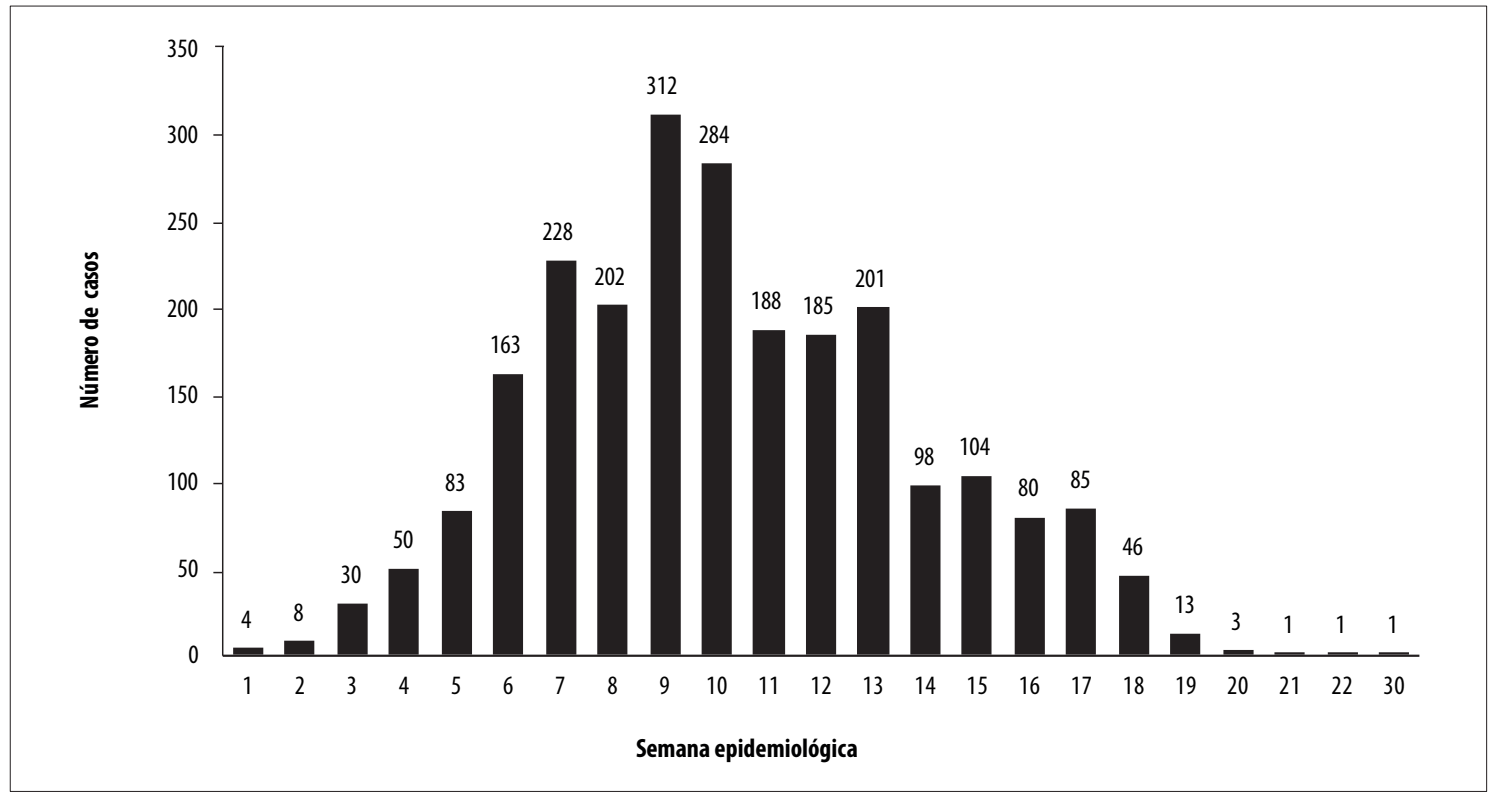

Figura 2 - Distribuição do número de casos confirmados de dengue por semana epidemiológica da notificação, Pinhalzinho, Santa Catarina, 2016

Em relação às variáveis climáticas, durante o período estudado, a temperatura mensal mínima variou de $-3,58$ a $16,04^{\circ} \mathrm{C}(\mathrm{DP} \pm 5,48)$, e a temperatura mensal máxima, de 26,65 a $36,48^{\circ} \mathrm{C}(\mathrm{DP} \pm 2,52)$; a temperatura média mensal variou entre 10,88 e $25,17^{\circ} \mathrm{C}$ $(\mathrm{DP} \pm 3,67)$. A precipitação mensal variou de 9,6 a 428mm (DP $\pm 107,18)$, e a umidade relativa do ar, entre 70,22 e $90,52 \%$ (DP $\pm 4,84)$. A umidade relativa e a temperatura média do ar apresentaram associação positiva - e significativa - com a infestação mensal pelo mosquito Ae. aegypti: $\mathrm{F}(2,39)=28,481$; valor $\mathrm{p}<0,001 ; \mathbf{R}^{2}=59,4 \%$ (Figura 3). 


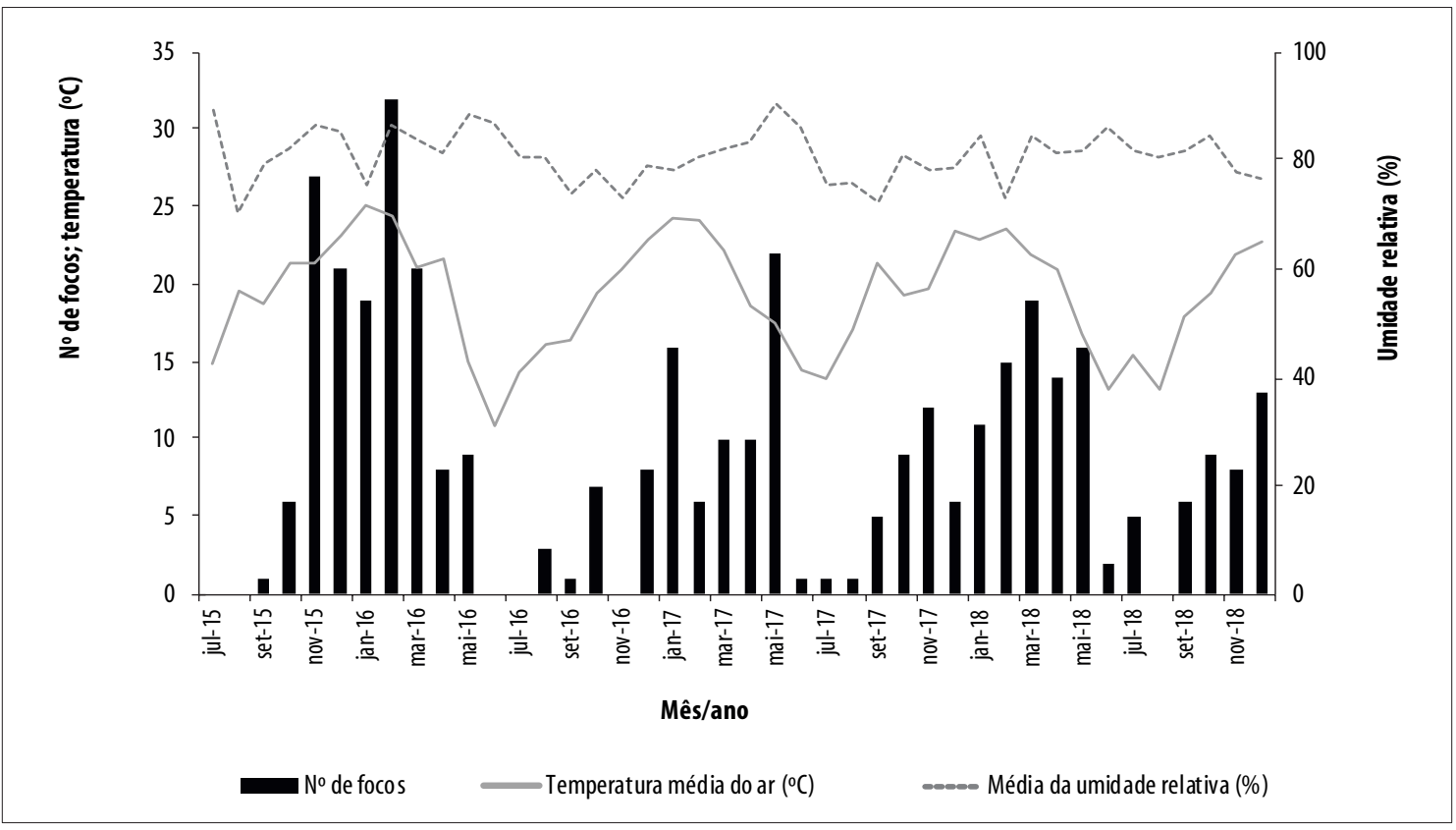

Figura 3 - Distribuição mensal do número de focos de Aedes aegyptiem comparação com a temperatura média mensal e a umidade relativa do ar, Pinhalzinho, Santa Catarina, 2015-2018

\section{Discussão}

Pinhalzinho enfrentou a maior epidemia de dengue já registrada no país, com taxa de incidência de 12.695,2 casos por 100 mil hab., causada pelo sorotipo DENV-1. Maiores taxas de incidência foram observadas em mulheres, entre pessoas com 50 anos de idade ou mais e no período de verão. A distribuição dos focos do mosquito revelou-se associada ao aumento da temperatura média e à umidade relativa do ar no município.

Essa epidemia demonstrou o aumento da expansão geográfica da doença e o processo de interiorização de sua transmissão, com registros de casos cada vez mais frequentes em pequenas cidades e no interior do país. ${ }^{4}$ Considerando-se que, a cada caso de dengue aparente, ocorrem outras quatro infecções inaparentes ou assintomáticas, ${ }^{1,2}$ estima-se que possivelmente cerca de $50 \%$ da população de Pinhalzinho tenha se infectado e sensibilizado pelo DENV-1. Uma alta incidência de dengue, como se observou em Pinhalzinho, geralmente ocorre quando as ações de vigilância e controle empregadas para prevenir a doença não são plenamente efetivas. ${ }^{78}$ Uma incidência tão alta, ademais, pode-se atribuir à elevada infestação pelo vetor Ae. aegypti e à introdução ou reintrodução de sorotipos do vírus em áreas onde há grande proporção de indivíduos susceptíveis.

Quanto ao sexo, o maior registro de casos em mulheres também foi observado em outros estudos. ${ }^{9-12}$ Este padrão pode estar relacionado à distribuição do Ae. aegypti, principalmente no intra no peridomicílio, ${ }^{13}$ ambientes mais frequentados por elas; ou também, ao fato de as mulheres procurarem mais pelos serviços de saúde e atendimento médico. ${ }^{9-11}$

Observou-se, outrossim, maior coeficiente de incidência nas idades mais avançadas, de 50 anos ou mais. Este achado diverge dos padrões relatados por outros estudos realizados no país, em que esse coeficiente é maior entre adultos jovens, particularmente na faixa etária entre 20 e 49 anos. ${ }^{9,10,12}$ Cumpre destacar que, embora todas as faixas etárias sejam igualmente suscetíveis, pessoas idosas têm maior risco de desenvolver dengue com sinais de alarme e dengue grave, possíveis de evoluir a óbito. ${ }^{4}$

A análise temporal apontou que os casos de dengue apresentaram um padrão sazonal de ocorrência: tiveram início nos meses de dezembro e janeiro, alcançando maior incidência em fevereiro e março, coincidindo com o período de temperaturas mais elevadas, $\mathrm{e}$ decréscimo no mês de maio. Padrões sazonais como 
este, de aumento na ocorrência de casos de dengue nos primeiros meses do ano, foram constatados em municípios dos estados de São Paulo,,${ }^{9,14,15}$ Piauí, ${ }^{16}$ Rio de Janeiro ${ }^{17}$ e Santa Catarina. ${ }^{18}$

Elevação da temperatura média e a umidade relativa do ar, juntas, foram capazes de prever $59,4 \%$ da infestação pelo vetor. Verifica-se que, nos dois anos após a epidemia, os focos de Aedes se mantiveram em níveis similares aos do período epidêmico, embora a transmissão do vírus tenha sido praticamente zerada.

A relação entre a precipitação pluviométrica e os focos de Ae. aegypti ou casos de dengue ocorre pela associação de diferentes mecanismos, ${ }^{19}$ podendo variar de acordo com a região estudada. A falta de associação significativa entre pluviosidade e número de focos de Ae. aegypti, observada neste estudo, pode ser explicada pela constância das chuvas ao longo do ano, na região.

Diante do exposto, cabe reforçar que 0 aumento da incidência e disseminação da dengue é um processo complexo, influenciado por múltiplos fatores sociais, ambientais e climáticos, os quais, de forma sinérgica, podem gerar um cenário epidêmico. ${ }^{17,20}$ Entre esses fatores, ressalta-se a urbanização desordenada, condições sanitárias precárias, falta de controle efetivo do mosquito, globalização da economia, propagação e infestação do vetor no ambiente urbano, mobilidade humana e mudanças climáticas. ${ }^{12,17-22}$

As principais limitações do presente estudo estão relacionadas às fontes de informações secundárias. A possibilidade de subnotificação e a existência de registros incompletos de casos de dengue podem comprometer a análise de algumas variáveis.

\section{Referências}

1. Bhatt S, Gething PW, Brady OJ, Messina JP, Farlow AW, Moyes CL, et al. The global distribution and burden of dengue. Nature [Internet]. 2013 Apr [cited 2019 May 17];496(7446):504-7. Available from: https://doi. org/10.1038/nature 12060

2. World Health Organization - WHO. Dengue control [Internet]. Geneva: World Health Organization; 2019 [cited 2019 Mar 12]. Available from: http://www.who. int/denguecontrol/en/

3. Valle D, Pimenta DN, Aguiar R. Zika, dengue e chikungunya: desafios e questões. Epidemiol Serv Saúde [Internet]. 2016 abr-jun [citado 2019 mar 26];25(2):419-22. Disponível em: http://dx.doi. org/10.5123/S1679-49742016000200020
Cabe mencionar que informações climáticas podem ser usadas para prever o aumento da população de mosquitos e, consequentemente, situar áreas de risco para surtos de arboviroses. Este alerta precoce pode ser usado pelas autoridades em Saúde Pública municipais, especialmente nos primeiros meses do ano, para planejar e direcionar medidas de maior eficácia no controle do mosquito Aedes e, assim, reduzir o risco de dengue e de outras arboviroses transmitidas por esse vetor.

0 conhecimento do processo epidêmico e o uso da informação podem auxiliar na avaliação da situação de saúde para a tomada de decisão, com vistas a direcionar ações intersetoriais, educativas e de conscientização social. Para reduzir o ônus da dengue, as políticas de Saúde Pública devem ser contínuas, e considerar necessidades locais específicas, no sentido do controle do vetor e da vigilância da doença. Finalmente, cumpre destacar a prioridade das ações de prevenção, com a participação ativa da população, articuladas com políticas públicas intersetoriais.

\section{Contribuição dos autores}

Andrioli DC, Busato MA e Lutinski JA contribuíram na concepção e delineamento do estudo, análise e interpretação dos resultados, redação e revisão crítica do conteúdo do manuscrito. Todos os autores aprovaram a versão final do manuscrito e declaram ser responsáveis por todos os seus aspectos, garantindo sua precisão e integridade.

4. Ministério da Saúde (BR). Secretaria de Vigilância em Saúde. Coordenação-Geral de desenvolvimento da Epidemiologia em Serviços. Guia de vigilância em saúde: volume único [Internet]. 2. ed. Brasília: Ministério da Saúde; 2017 [citado 2019 maio 17]. 705 p. Disponível em: http://portalarquivos.saude.gov.br/ images/pdf/2017/outubro/06/Volume-Unico-2017.pdf

5. Estado de Santa Catarina. Secretaria de Estado da Saúde. Diretoria de Vigilância Epidemiológica. Plano de contingência para o enfrentamento da dengue, febre de chikungunya e vírus Zika no estado de Santa Catarina [Internet]. Florianópolis: Secretaria de Estado da Saúde; 2017 [citado 2019 abr 4]. 16 p. Disponível em: http://www.dive.sc.gov.br/conteudos/ publicacoes/PlanoContingenciadengue.pdf 
6. Instituto Brasileiro de Geografia e Estatística - IBGE. Pesquisa de informações básicas municipais: Pinhalzinho [Internet]. Rio de Janeiro: Instituto Brasileiro de Geografia e Estatística; 2017 [citado 2019 fev 26]. Disponível em: http://cidades.ibge.gov. $\mathrm{br} / \mathrm{brasil} / \mathrm{sc} /$ pinhalzinho/panorama

7. Valle D, Bellinato DF, Viana-Medeiros PF, Lima JBP, Martins Junior ADJ. Resistance to temephos and deltamethrin in Aedes aegypti from Brazil between 1985 and 2017. Mem Inst Oswaldo Cruz [Internet]. 2019 [cited 2019 Jun 10];114:e180544. Available from: https://doi.org/10.1590/0074-02760180544

8. Morato DG, Barreto FR, Braga JU, Natividade MS, Costa MCN, Morato V, et al. The spatiotemporal trajectory of a dengue epidemic in a medium-sized city. Mem Inst Oswaldo Cruz [Internet]. 2015 Jun [cited 2019 Jun 11];110(4):528-33. Available from: https://doi. org/10.1590/0074-0276140388

9. Ferreira AC, Chiaravalloti Neto F, Mondini A. Dengue em Araraquara, SP: epidemiologia, clima e infestação por Aedes aegypti. Rev Saúde Pública [Internet]. 2018 fev [citado 2019 jun 18];52:18. Disponível em: https://doi.org/10.11606/s1518-8787.2018052000414

10. Oliveira RMAB, Araújo FMC, Cavalcanti LPG. Aspectos entomológicos e epidemiológicos das epidemias de dengue em Fortaleza, Ceará, 2001-2012. Epidemiol Serv Saúde [Internet]. 2018 [citado 2019 jun 18];27(1):e201704414. Disponível em: https://doi. org/10.5123/s1679-49742018000100014

11. Rodrigues NCP, Daumas RP, Almeida AS, Santos RS, Koster I, Rodrigues PP, et al. Risk factors for arbovirus infections in a low-income community of Rio de Janeiro, Brazil, 2015-2016. PloS One [Internet]. 2018 Jun [cited 2019 Jun 18];13(6):e0198357. Available from: https://doi.org/10.1371/journal.pone.0198357

12. San Martín JL, Brathwaite 0, Zambrano B, Solórzano J0, Bouckenooghe A, Dayan GH, et al. The epidemiology of dengue in the Americas over the last three decades: a worrisome reality. Am J Trop Med Hyg [Internet]. 2010 Jan [cited 2019 Jun 20];82(1):12835. Available from: https://doi.org/10.4269/ ajtmh.2010.09-0346

13. Ministério da Saúde (BR). Secretaria de Vigilância em Saúde. Saúde Brasil 2015/2016: uma análise da situação de saúde e da epidemia pelo vírus Zika e por outras doenças transmitidas pelo Aedes aegypti [Internet]. Brasília: Ministério da Saúde; 2017 [citado 2019 maio 22]. 386 p. Disponível em: http://bvsms.saude.gov.br/bvs/publicacoes/saude brasil_2015_2016_analise_zika.pdf

14. Chiaravalloti-Neto F, Pereira M, Fávaro EA, Dibo MR, Mondini A, Rodrigues-Junior AL, et al. Assessment of the relationship between entomologic indicators of Aedes aegypti and the epidemic occurrence of dengue virus 3 in a susceptible population, São José do Rio Preto, São Paulo, Brazil. Acta Trop [Internet]. 2015 Feb [cited 2019 Jun 28];142:167-77. Available from: https://doi.org/10.1016/j.actatropica.2014.11.017

15. Carneiro MAF, Alves BCA, Gehrke FS, Domingues JN, Sá N, Paixão S, et al. Environmental factors can influence dengue reported cases. Rev Assoc Med Bras [Internet]. 2017 Nov [cited $2020 \mathrm{Apr}$ 02];63(11):957-61. Available from: https://doi. org/10.1590/1806-9282.63.11.957

16. Valladares GS, Hassum IC, Albuquerque ELS, Santos AC. Influência de variáveis ambientais na ocorrência da dengue utilizando geoprocessamento em Teresina, Piauí. Hygeia [Internet]. $2019 \mathrm{dez}$ [citado $2020 \mathrm{abr}$ 1];15(34):102-14. Disponível em: http://dx.doi. org/10.14393/Hygeia153447217

17. Xavier DR, Magalhães MAFM, Gracie R, Reis IC, Matos VP, Barcellos C. Difusão espaço-tempo do dengue no Município do Rio de Janeiro, Brasil, no período de 2000-2013. Cad Saúde Pública [Internet]. 2017 mar [citado 2019 jun 23];33(2):e00186615. Disponível em: https://doi. org/10.1590/0102-311x00186615

18. Busato MA, Machado CS, Lutinski JA, Shabat FM, Simões DA, Siebel AM, et al. Environmental factors associated to dengue fever occurrence in the Chapecó municipality, Santa Catarina State. Semina [Internet]. 2019 Jul-Dec [cited $2020 \mathrm{Apr}$ 01];40(2):237-48. Available from: https://dx.doi. org/10.5433/1679-0367.2019v40n2p237

19. Lowe R, Gasparrini A, Van Meerbeeck CJ, Lippi CA, Mahon R, Trotman AR, et al. Nonlinear and delayed impacts of climate on dengue risk in Barbados: a modelling study. PLoS Med [Internet]. 2018 Jul [cited 2019 Jun 26];15(7):e1002613. Available from: https:// dx.doi.org/10.1371\%2Fjournal.pmed.1002613

20. Gubler DJ. Dengue, urbanization and globalization: the unholy trinity of the 21st century. Trop Med Health [Internet]. 
2011 Dec [cited 2019 Jun 27];39(4

Suppl):3-S11. Available from: https://dx.doi. org/10.2149\%2Ftmh.2011-S05

21. Caldas E, Santos DM, Gallas M, Silveira EF, Périco E. Influência de determinantes ambientais e socioeconômicos nos casos de dengue na cidade de Porto Alegre, RS. In: Anais XVII Simpósio Brasileiro de Sensoriamento Remoto - SBSR;2015 abr 25-29
abr;João Pessoa (PB), Brasil. João Pessoa: INPE; 2015 [citado 2020 jun 10]. p. 2182-89. Disponível em: http://www.dsr.inpe.br/sbsr2015/files/p0443.pdf

22. Sun W, Xue L, Xie X. Spatial-temporal distribution of dengue and climate characteristics for two clusters in Sri Lanka from 2012 to 2016. Sci Rep [Internet]. 2017 Oct [cited 2019 Jun 27];7(12884). Available from: https://doi.org/10.1038/s41598-017-13163-Z

\begin{abstract}
Objective: To describe the characteristics of the dengue epidemic in Pinhalzinho, Santa Catarina, Brazil, and to investigate the effects of climate variables on Aedes aegypti mosquito infestation. Methods: This was an ecological study using data on dengue cases, hospitalizations and deaths in 2015 and 2016; in addition to climate variables and Aedes aegypti breeding grounds from 2015 to 2018. Results: In the 2015-2016 epidemic, the dengue incidence rate was 12,695.2/100,000 inhabitants. Higher incidence was registered in the female sex (13,926.4/100,000 inhabitants) and in the 50 years and over age group $(17,162.0 / 100,000$ inhabitants). Average temperature and relative humidity showed a positive relationship with increase in Aedes aegypti breeding grounds. Conclusion: Dengue incidence during the epidemic was the highest ever recorded in the country. Climate conditions must be considered when planning vector control and dengue prevention actions.

Keywords: Aedes; Climate; Epidemiology, Descriptive.
\end{abstract}

\begin{abstract}
Resumen
Objetivo: Analizar las características de la epidemia de dengue en Pinhalzinho, Santa Catarina, Brasil, y evaluar los efectos de las variables climáticas en la infestación del mosquito Aedes aegypti. Métodos: Estudio ecológico con datos sobre casos de dengue, hospitalizaciones y óbitos en 2015 y 2016, además de las variables meteorológicas y focos de Ae. Aegypti de 2015 a 2018. Resultados: En el período epidémico de 2015-2016, la incidencia de dengue fue 12.695,2 casos/100 mil habitantes. Se registró la mayor incidencia en el sexo femenino y en el grupo de edad superior a los 50 años. El promedio de temperatura y humedad relativa del aire presentaron una relación positiva con el aumento de los brotes de Ae. aegypti. Conclusión: La epidemia fue la más grande registrada en el país. La evaluación del proceso epidémico y las condiciones climáticas se constituyen en importante berramienta para planificación del control vectorialy acciones de prevención al dengue.
\end{abstract}

Palabras clave: Aedes; Clima; Epidemiología Descriptiva.

Recebido em 21/02/2020

Aprovado em 22/05/2020

Editora associada: Luciana Guerra Gallo - ํ orcid.org/0000-0001-8344-9951 